\title{
Emergency physician-performed ultrasound-guided nerve blocks in proximal femoral fractures provide safe and effective pain relief: a prospective observational study in The Netherlands
}

\author{
Rein Ketelaars ${ }^{1 *}$ (D), Joram T. Stollman ${ }^{2,3}$, Evelien van Eeten ${ }^{2}$, Ties Eikendal ${ }^{2}$, Jörgen Bruhn ${ }^{1}$ and Geert-Jan van Geffen ${ }^{1}$
}

\begin{abstract}
Background: The treatment of acute pain in the emergency department is not always optimal. Peripheral nerve blocks using "blind" or nerve stimulator techniques have substantial disadvantages. Ultrasound-guided regional anesthesia may provide quick, safe, and effective pain relief in patients with proximal femoral fractures with severe pain. However, no evidence exists on emergency physician-performed ultrasound-guided regional anesthesia in these patients in Dutch emergency departments. We hypothesized that emergency physicians can be effectively trained to safely perform and implement ultrasound-guided femoral nerve blocks, resulting in effective pain relief in patients with proximal femoral fractures.

Methods: In this prospective observational study, emergency physicians were trained by expert anesthesiologists to perform ultrasound-guided femoral nerve blocks during a single-day course. Femoral nerve blocks were performed on patients with proximal femoral fractures. A system of direct supervision by skilled anesthesiologists and residents was put in place.

Results: A total of 64 femoral nerve blocks were performed. After 30 min, blocks were effective in $69 \%$ of patients, and after $60 \mathrm{~min}$, in $83.3 \%$. The mean reduction in pain scores after 30 and 60 min was 3.84 and 4.77, respectively (both $p<0.001$ ).

Patients reported a mean satisfaction of 8.42 (1 to 10 scale). No adverse events occurred.

Conclusions: Ultrasound-guided femoral nerve block is an effective, safe, and easy to learn (single-day course) procedure for emergency physicians to implement and perform in the emergency department. Patient satisfaction was high.
\end{abstract}

\section{Background}

The treatment of acute pain in emergency department (ED) patients is not always optimal [1, 2]. Fortunately, in patients with proximal femoral fractures, peripheral nerve blocks are used increasingly to obtain adequate pain relief [3-6]. In addition to providing pain relief, it may decrease the administration of systemic analgesics

\footnotetext{
* Correspondence: Rein.Ketelaars@radboudumc.nl; rein@ketelaars.nu 'Department of Anesthesiology, Pain and Palliative medicine, Radboud university medical center, Geert Grooteplein-Zuid 10, 6525 GA Nijmegen, The Netherlands

Full list of author information is available at the end of the article
}

such as opioids and decrease their side-effects [7, 8]. Also, undertreated pain and inadequate analgesia have the potential to cause delirium in patients with proximal femoral fractures [9]. Fascia iliaca compartment blocks (FICB) have been performed in hip surgery patients and have shown the potential to reduce the incidence of perioperative delirium in these patients [10].

Nerve blocks in femoral neck fracture patients can be achieved using different techniques. The FICB is a "blind" technique in which surface anatomy landmarks are used to determine the needle insertion point, and 
tactile feedback guides the correct needle position. Techniques based on surface landmarks have a higher incidence of paresthesia during performance of the block [11]. Furthermore, they produce blocks with a slower onset, lower quality, and shorter duration compared to an ultrasound-guided technique $[4,11,12]$. A nerve stimulator-guided femoral nerve block makes use of electrical nerve stimulation to locate the femoral nerve. If a minimal electrical current still elicits quadriceps muscle contractions, the optimal needle tip position is obtained. Especially in proximal femoral fracture patients, these contractions may be painful and are therefore undesirable [4].

Ultrasound-guided nerve blocks may overcome the aforementioned drawbacks. Ultrasonography allows identification of relevant anatomical structures and continuous needle tip visualization, and even the spread of local anesthetic (LA) may be observed. Ultrasonography in regional anesthesia has increased the success rate and reduced the complications of peripheral nerve blocks [13].

Traditionally, only anesthesiologists performed ultrasound-guided regional anesthesia (UGRA). In recent years, though, emergency physicians (EPs) have been adopting this technique [14-16]. However, in The Netherlands, a lack of evidence exists on EP-performed UGRA in proximal femoral fracture patients.

An ultrasound-guided femoral nerve block and FICB appears to provide quick, safe, and effective acute pain relief and could therefore be a valuable tool adding to current pain management regimes in Dutch EDs [7, 17]. However, EPs should gain relevant knowledge on basic ultrasonography, local anesthetics, nerve block indications, relevant anatomy, block techniques, and complications. Relevant skills to acquire are ultrasound scanning techniques, recognizing sonoanatomy, and ultrasoundguided needle handling. We hypothesize that EPs can be effectively trained to safely perform ultrasound-guided femoral nerve blocks, resulting in effective pain relief in patients with proximal femoral fractures.

\section{Methods}

Design

A prospective observational study in ED patients with proximal femoral fractures was conducted from June 2014 until June 2017. The aim of the study was to evaluate the effectiveness, safety, and satisfaction of EP-performed ultrasound-guided nerve blocks in the emergency department.

\section{Recruitment and setting}

Adult patients admitted to the Radboud university medical center ED with a proximal femoral fracture, including trochanteric and femoral neck fractures, in whom an ultrasound-guided femoral nerve block or FICB was planned in the ED were included. Exclusion criteria were any sign of infection at the injection site, hemorrhagic diathesis (e.g., hemophilia and use of anticoagulant drugs with international normalized ratio (INR) $>2.0$ ), an allergy to ropivacaine, and multiple traumata.

A 1-day course and an e-learning module were developed by a collaboration of anesthesiologists with extensive experience in UGRA and EPs experienced in general ultrasound. Every EP and EP in training participated in this hands-on course focusing on UGRA of the femoral nerve and FICB. The online pre-course e-learning module and course lectures dealt with basic theory of ultrasound, pharmacology of local anesthetics, indications, relevant anatomy, block techniques, complications and their treatment, and follow-up. Live anatomy and practical block techniques were taught on human cadavers at the Radboud Anatomy Department. Scanning techniques and sonoanatomy were taught and practiced on the participants themselves. Ultrasound-guided needle handling was practiced on blocks of tofu with tiny artifacts inserted.

The UGRA-trained EPs were supervised by skilled (resident) anesthesiologists for five, or more if desired, ultrasound-guided femoral nerve blocks or FICBs until the EPs had gained the knowledge, expertise (as judged by the anesthesiologist), and confidence to perform the procedure independently. A dedicated pool of anesthesiologists and residents with extensive experience in UGRA provided immediate and direct supervision on weekdays during regular business hours. Outside of these hours, supervision was provided depending on the availability of a skilled anesthesiologist or resident.

Before the ultrasound-guided femoral nerve block or FICB was performed, oral informed consent was obtained as part of the standard operating procedure (SOP). No sedative premedication was administered prior to the procedure.

The ED SOP was jointly written by anesthesiologists and EPs, in accordance with the existing SOP for ultrasound-guided femoral nerve blocks performed by anesthesiologists in the operating room. It prescribes a sterile procedure and a conservative maximal LA dosage to minimize risks of local anesthetic systemic toxicity (LAST). Vital parameters (continuous electrocardiogram, pulse rate, oxygen saturation, respiratory rate, and blood pressure) are monitored and continued for $30 \mathrm{~min}$ after the procedure. The skin is prepped and draped, and face masks, caps, sterile gloves, and a sterile probe cover are donned. Sterile ultrasound transmission gel and a Stimuplex ${ }^{\ominus}$ Ultra $22 \mathrm{G} 0.64 \times 50 \mathrm{~mm}, 30^{\circ}$, short bevel (B. Braun, Melsungen, Germany) non-traumatic needle are used. The LA consists of ropivacaine $0.375 \%$, in (four) labeled 10-ml syringes, to allow for a high-volume block without exceeding the maximum dosage. Alternatively, ropivacaine $0.75 \%$, which is also used by 
anesthesiologists for providing surgical pain relief, can be used. The maximum allowed dose of LA is $2 \mathrm{mg} \cdot \mathrm{kg}^{-1}$ body weight of ropivacaine, injected in 1-2 $\mathrm{ml}$ increments under direct ultrasound guidance to confirm the optimal spread of LA around the femoral nerve or below the iliac fascia (FICB). Negative aspiration is confirmed at least every $5 \mathrm{ml}$ to prevent intravascular injection.

In addition to the EPs and EPs in training, all ED nurses were trained to be familiar with the SOP and to be able to assist in the procedures.

The procedure was recorded in the electronic medical record (EMR) according to the SOP. Relevant and additional data were recorded on a dedicated case report form, including gender, age, fracture type and location, and prehospitally administered analgesics (type, dosage, and route of administration). The indication and type of the nerve block, LA dosage, scores on physician and patient satisfaction, pain scores, any rescue medication, adverse events, and any reason to abandon the procedure were also recorded. Vital signs were recorded only in the EMR.

Pain scores were taken in rest on a numeric rating scale (NRS; 0 to 10 ) on arrival at the ED (t0) and after the procedure at 30,60 , and $120 \mathrm{~min}$ ( $\mathrm{t} 30, \mathrm{t} 60$, and $\mathrm{t} 120$, respectively), but only if still in the ED. We considered a nerve block to be successful whenever there was a pain reduction of at least two points after 30 min compared to baseline. We considered a pain reduction of at least $33 \%$ to be clinically important, as inspired by the work of Farrar et al. [18]. An absolute pain score of 4 or less, though, was considered acceptable pain.

Just before discharge from the ED, patients were asked about the level of (dis)comfort they experienced and if they were motivated to undergo a similar procedure in the future. In addition, EPs self-reported five attributes of the procedure. These attributes were scored on a 1-10 rating scale as shown in Table 1.

\section{Statistical analysis}

Normally distributed data are reported as mean \pm standard deviation (SD) or $95 \%$ confidence interval (CI), and data with a skewed distribution, including absolute pain scores, are reported as median with an interquartile range (IQR). A one-tailed paired Wilcoxon test was used to test for differences in pain scores within subjects because these are ordinal values, not normally distributed. To test for differences between subgroups in relative and absolute pain score changes (normally distributed), a $t$ test and a one-way ANOVA was used. Pearson correlation was calculated for the influence of age and injected volume of LA. Statistical significance was considered at $P<0.05$. For statistical analysis, IBM SPSS Statistics for Windows, version 22.0 (IBM Corp., Armonk, NY, USA), and GraphPad Prism version 5.00 for Windows (GraphPad Software, San Diego, CA, USA) were used.

\section{Results and discussion}

In total, 64 patients (23 males, 41 females) received UGRA by EPs in the ED for a proximal femoral fracture. Demographics, types of fractures, and prehospitally administered analgesics are displayed in Table 2.

Fourteen EPs and EPs in training performed on average 4.6 (CI 3.1-6.0) ultrasound-guided nerve blocks of which 13 (20.3\%) were FICBs and 51 (79.7\%) femoral nerve blocks.

Ropivacaine $0.375 \%$ was used in $28(43.8 \%)$ patients with a mean volume of $22.8 \mathrm{ml}( \pm 6.6 \mathrm{ml}$, range 13 $40 \mathrm{ml}$ ). Ropivacaine $0.75 \%$ was used in $36(56.3 \%)$ patients with a mean volume of $18.9 \mathrm{ml}( \pm 2.9 \mathrm{ml}$, range $10-20 \mathrm{ml}$ ). All blocks proceeded uneventfully, although one patient with mild pain on injection and one event of transient periprocedural hypotension, unrelated to LA injection, were reported.

\section{Patient perspective}

The median pain score on an NRS at baseline (t0) before UGRA was 8 (IQR 5-9). At $30 \mathrm{~min}$ (t30) after the nerve

Table 1 Attributes of the ultrasound-guided regional anesthesia procedure

\begin{tabular}{lll}
\hline Attribute & Score-1 & Score-10 \\
\hline $\begin{array}{l}\text { Patient } \\
\text { (Dis)comfort experienced during the procedure }\end{array}$ & Very uncomfortable & Not uncomfortable at all \\
Would like to undergo a similar procedure in the future & Would like it never again & Would like it again \\
$\begin{array}{l}\text { Emergency physician } \\
\text { Ease of procedure }\end{array}$ & Very difficult & Very easy \\
Success of procedure itself regardless of the effect & Did not succeed at all & Very successful procedure \\
Visibility of anatomical structures on ultrasound & Hard to recognize & Easy to recognize \\
Spread of local anesthetic on ultrasound & Bad spread & Good spread \\
Subjective added value of procedure to patient care & No added value & Absolute added value to patient care \\
\hline
\end{tabular}

These attributes were to be reported by the patients and self-reported emergency physicians on a 1-10 numeric rating scale 
Table 2 Demographics and type of fracture

\begin{tabular}{|c|c|c|c|}
\hline Factors & Frequency or value & $\%$ & Range \\
\hline Median age (IQR), year & $76(68-84)$ & & $28-95$ \\
\hline \multicolumn{4}{|l|}{ Gender } \\
\hline Male & 23 & 35.9 & \\
\hline Female & 41 & 64.1 & \\
\hline \multicolumn{4}{|l|}{ Type of fracture } \\
\hline Femoral neck & 37 & 57.8 & \\
\hline Trochanteric & 16 & 25.0 & \\
\hline Femoral shaft & 11 & 17.2 & \\
\hline \multicolumn{4}{|l|}{ Laterality } \\
\hline Left femur & 30 & 46.9 & \\
\hline Right femur & 34 & 53.1 & \\
\hline \multicolumn{4}{|l|}{ Prehospital analgesics } \\
\hline Rate of administration & 51 & 79.7 & \\
\hline Paracetamol/acetaminophen & 19 & 37.3 & \\
\hline NSAIDs & 0 & 0 & \\
\hline Oxycodone & 1 & 2.0 & \\
\hline Fentanyl & 26 & 51.0 & \\
\hline Morphine & 6 & 11.8 & \\
\hline Esketamine & 14 & 27.5 & \\
\hline
\end{tabular}

IQR interquartile range, NSAIDs nonsteroidal anti-inflammatory drugs

block, the median score was significantly reduced to 3 (IQR $2-5, P<0.001$ ). The pain was significantly reduced even further to 2 (IQR $0-4$ ) at t60 and to 1 (IQR $0-1$ ) at t120. Relative and absolute pain reductions are shown in Table 3 and Fig. 1.

Patient-reported (dis)comfort was scored a median of 8 (IQR $8-9, n=61$ ). When asked if they were motivated to undergo a similar procedure in the future, the score was 9 (IQR $8-10, n=60)$.

Pain reduction was not found to be significantly related to gender, age, fracture types, prehospital administration (and type) of analgesics, the two block types, the two different concentrations of ropivacaine, or volume of injected LA. Also, we found no significant difference in pain reduction at $\mathrm{t} 30$, t60, and $\mathrm{t} 120$, whether the EPs performed the blocks with or without supervision. As shown in Table 4.

\section{EP perspective}

The 1-day course was very well received, and the EPs were enthusiastic about starting to perform the nerve blocks after the course. The median scores, IQR in parentheses, of the self-reported qualifications of the procedure $(n=59)$ were ease of procedure $8(7-9)$, success of procedure itself, regardless of the effect $9(8-10)$, visibility of anatomical structures on ultrasound 8 (7-9), spread of local anesthetic 9 (8-10), and subjective added value of the procedure to patient care $9(8-10)$.

\section{Discussion}

We showed that, after having received appropriate training, Dutch EPs are able to safely perform UGRA in ED patients presenting with a proximal femoral fracture and severe pain. After 30 and $60 \mathrm{~min}$, mean pain reduction was respectively 3.84 (50.9\%) and 4.77 (64.4\%). A pain score of 4 or less was reported by 69 and $83.3 \%$, respectively, of the patients. The EPs thought the procedure was easy to perform, and they were able to obtain a good visualization of the relevant anatomy and LA spread. This means that performing a peripheral nerve block is an effective pain relief strategy provided by the very first physician they encounter upon admittance in the hospital.

To date, there are only few similar reports of EPs performing UGRA in the ED. At 30 and $60 \mathrm{~min}$ after the nerve block, we found a meaningful pain reduction, respectively, in 70.7 and $80.0 \%$ of the patients. We could confirm the results reported by Groot et al. who reported that on a Dutch ED, EP-performed blind FICBs were safe and effective. In 26 of 34 patients (76\%), they found a clinically meaningful pain score reduction after 120 min [19]. An explanation for the slightly better and faster pain reduction in the present study might be that we have used an ultrasound-guided technique, compared to their blind FICB. Accurate deposition of the LA in

Table 3 Pain scores and reduction from baseline at 30, 60, and 120 min

\begin{tabular}{|c|c|c|c|c|c|c|c|}
\hline & $N(\%)$ & $\begin{array}{l}\text { Pain score, } \\
\text { median (IQR) }\end{array}$ & $\begin{array}{l}\text { Pain score } \\
\leq 4 n(\%)\end{array}$ & $\begin{array}{l}\text { Pain reduction } \\
\geq 33 \% n(\%)\end{array}$ & Pain reduction & $\mathrm{Cl}$ & $p$ value \\
\hline Baseline & $64(100)$ & $8(5-9)$ & $6(9.4 \%)$ & - & - & & \\
\hline \multirow[t]{2}{*}{ t30 } & $58(90.6)$ & $3(2-5)$ & $40(69.0)$ & $41(70.7)$ & 3.84 & $3.15-4.54$ & $<0.001$ \\
\hline & & & & & $50.9 \%$ & $42.6-59.2$ & $<0.001$ \\
\hline \multirow[t]{2}{*}{ t60 } & $30(46.9)$ & $2(0-4)$ & 25 (83.3) & $24(80.0)$ & 4.77 & $3.73-5.80$ & $<0.001$ \\
\hline & & & & & $64.4 \%$ & $52.1-76.8$ & $<0.001$ \\
\hline \multirow[t]{2}{*}{ t120 } & $7(10.9)$ & $1(0-1)$ & $6(85.7)$ & $6(85.7)$ & 5.85 & $2.72-8.99$ & 0.002 \\
\hline & & & & & $79.5 \%$ & $46.3-100.0$ & $<0.001$ \\
\hline
\end{tabular}

Median pain scores at baseline, 30,60, and 120 min. Pain scores of 4 or lower, pain reduction within subjects of at least $33 \%$, overall pain reduction relative, and in numeric rating scale (NRS) points including $95 \%$ confidence interval $(\mathrm{Cl})$ and $p$ value 


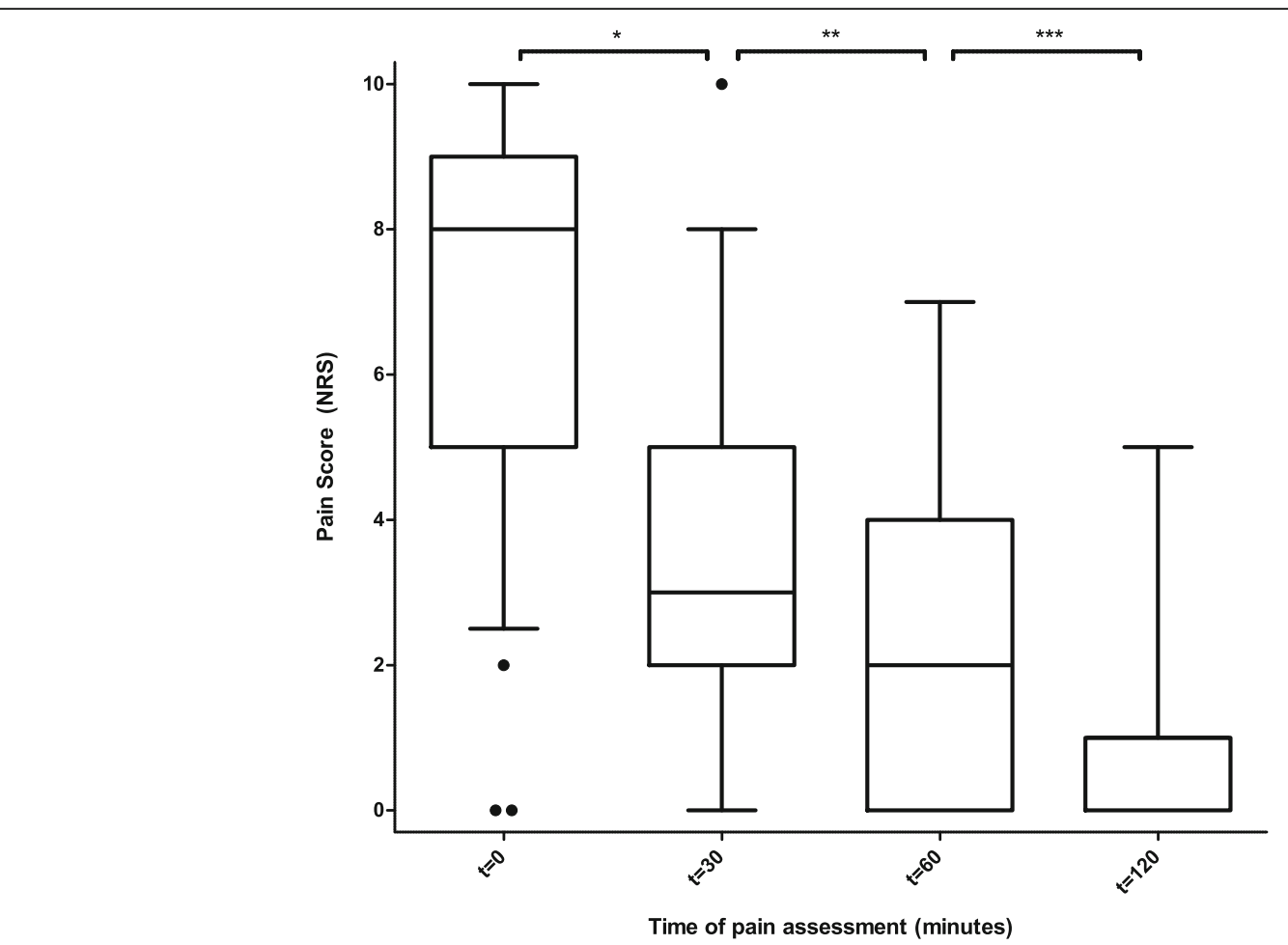

Fig. 1 Pain scores at baseline and after emergency department ultrasound-guided regional anesthesia in proximal femoral fractures. Pain scores at baseline and at 30, 60, and 120 min after an emergency physician-performed ultrasound-guided nerve block in emergency department patients with a proximal femoral fracture. 0 = absolutely no pain; $10=$ most extreme pain. Boxes show median and interquartile range, whiskers mark the minimum and maximum $\left(1.5 \times\right.$ lower and upper quartile), and dots are outliers. NRS numeric rating scale. ${ }^{*} p<0.001 ;{ }^{* *} p<0.001 ;{ }^{* *} p=0.03$

Table 4 Influence of relevant factors on absolute pain reduction 30 min after ultrasound-guided regional anesthesia

\begin{tabular}{|c|c|c|c|}
\hline Factors & Mean difference & $95 \% \mathrm{Cl}$ & $p$ value \\
\hline \multicolumn{4}{|l|}{$t$ test } \\
\hline Gender (male, female) & -0.03 & $-1.48-1.42$ & 0.97 \\
\hline Laterality (left, right) & -0.55 & $-1.95-0.85$ & 0.43 \\
\hline Prehospital analgesics (no, yes) & 0.96 & $-3.56-5.47$ & 0.57 \\
\hline Block type (femoral nerve, FICB) & 1.25 & $-0.32-2.81$ & 0.11 \\
\hline Ropivacaine concentration $(0.375 \%, 0.75 \%)$ & -0.13 & $-1.56-1.30$ & 0.43 \\
\hline \multicolumn{4}{|l|}{ One-way ANOVA } \\
\hline $\begin{array}{l}\text { Fracture type (femoral neck, trochanteric, } \\
\text { femoral shaft) }\end{array}$ & & & 0.10 \\
\hline Correlation & Pearson's $r$ & & \\
\hline Age & -0.063 & & 0.32 \\
\hline Volume of ropivacaine $0.375 \%$ & -0.230 & & 0.14 \\
\hline Volume of ropivacaine $0.75 \%$ & -0.078 & & 0.33 \\
\hline
\end{tabular}

Mean difference is without dimension because it is the absolute reduction in pain score on a $0-10$ numeric rating scale. A positive difference indicates the pain score reduction is larger in a variable's second value

FICB fascia iliaca compartment block 
relation to the fascia iliaca, or adjacent to or surrounding the femoral nerve will lead to a more effective and faster effect.

Dochez et al. described the effect of blind FICBs performed by EMS nurses in 100 patients with suspected proximal femoral fractures. After $30 \mathrm{~min}$, they reported a successful block in $96 \%$ and median pain scores were reduced from 8 to 3, and on arrival at the ED, 75\% had a pain score of 4 or less [20]. Median pain reduction at $t=30$ and pain scores of 4 or less were comparable (respectively, 5 and 69.9\%).

Gozlan et al. described prehospital EP-performed blind FICBs in 52 patients with femoral fractures and reported a success rate of $94 \%$ and pain reduction comparable to Dochez and the present study [21]. Morrison et al. compared standard analgesics with EP-performed ultrasound-guided femoral nerve blocks and found a significant difference in favor of the latter. Baseline NRS was 6.4 in both groups and decreased to 5.3 and 3.5, respectively, after $2 \mathrm{~h}$. Unfortunately, they did not report within-subject pain score reduction in the nerve block group [15].

A study by Beaudoin et al. reported similar results to our study [17]. Thirty-six ED patients with proximal femoral fractures were randomized between a femoral nerve block and conventional analgesia. The median pain score in the nerve block group reduced from a mean NRS of 8 at baseline to 4 after $4 \mathrm{~h}$. In the group receiving standard care, the NRS was 8 initially and showed no improvement [17]. Haines et al. described 20 ED ultrasound-guided FICBs performed by six EPs, fellows, and residents. They found a pain reduction from a mean NRS of 7.9 at baseline to 2.05 at $30 \mathrm{~min}$ and 1.30 at $120 \mathrm{~min}$ [22].

We showed EPs and EPs in training can be taught to effectively and safely perform UGRA in the ED. In addition, they experienced the procedure to be relatively easy and successful, represented by the high EP-reported scores for success, easiness, visibility of the anatomy, and quality of LA spread. These findings implicate that (Dutch) EPs should consider the introduction of ultrasound-guided regional anesthesia in proximal femoral fracture patients in the ED to provide superior pain management as compared to conventional systemic analgesics. Such a program should preferably be introduced in cooperation with their colleagues from the anesthesiology department. Once a successful UGRA program for these patients is implemented, it can be extended to other indications in need of excellent pain management.

With this project, we followed the recommendation by $\mathrm{Wu}$ et al. that anesthesiologists with extensive experience in regional anesthesia should introduce these techniques into settings outside the operating room and in the early treatment phases of trauma patients to provide the benefits of regional anesthesia [23]. Although in our institution, skilled anesthesiologists collaborated with EPs to successfully introduce UGRA in the ED, this approach might not be feasible in other comparable hospitals. EPs must connect with instructors with sufficient skills who are willing and able to invest their time and energy. Nevertheless, superior pain relief should be obtained in trauma patients as early as possible, preferably by the first-prehospital-care provider they encounter [24]. If this journey cannot start at home, adequate pain relief needs to be taken care of by the first care provider they encounter in the hospital.

This study adds to the literature because we took a unique approach in the introduction and execution of these blocks on our ED through a productive collaboration between the two (ED and anesthesiology) departments.

Furthermore, to date, there have been no published reports of effective and safe UGRA in a Dutch ED.

\section{Limitations}

This study has several limitations. The observational study design is not optimal to answer the main question. A relatively small number of patients was included based on convenience sampling. The aim was to treat proximal femoral fracture patients with ultrasound-guided nerve blocks as the treatment of choice. Although UGRA was recently introduced in our ED, its effectiveness has been proven extensively $[8,13,25]$. To guarantee efficacy and safety of the blocks in the ED setting, anesthesiologists have trained the EPs and supervised the blocks on request. Therefore, we chose not to compare with traditional treatment strategies but to investigate pain reduction within subjects and explore subjective experiences of both health care providers and patients. Another limitation is the increasing amount of missing data at the 30-, 60-, and 120min intervals from baseline. This might be caused by high ED work-load, resulting in incompletely filled-out case report forms, but is mainly due to expeditious patient transfer to the ward or the operating room. Also, there is a risk of bias because the EPs and ED nurses who performed the blocks filled out the case report forms themselves. These limitations can be partly justified because the efficacy of these blocks has been proven in general. In the present study, they have been performed in most (78\%) cases by at least two physicians (or residents) and an ED nurse all verifying the correct technique.

\section{Conclusions}

In conclusion, this study demonstrates that, through close cooperation between EPs and anesthesiologists, after a 1-day training (Dutch), EPs can learn to safely perform ultrasound-guided nerve blocks in proximal femoral fracture patients in the ED, resulting in effective acute pain relief. 


\section{Abbreviations}

ANOVA: Analysis of variance; Cl: Confidence interval; ED: Emergency department; EMR: Electronic medical record; EP: Emergency physician; FICB: Fascia iliaca compartment block; IQR: Interquartile range; LA: Local anesthetic; LAST: Local anesthetic systemic toxicity; NRS: Numeric rating scale; NSAID: Nonsteroidal anti-inflammatory drug; SD: Standard deviation; SOP: Standard operating procedure; UGRA: Ultrasound-guided regional anesthesia

\section{Acknowledgements}

We would like to thank all emergency physicians and residents of the Radboud university medical center for participating in the project and their contribution to this paper. We thank Prof. G.J. Scheffer for providing comments on an earlier version of the manuscript.

\section{Funding}

No funding was received.

\section{Availability of data and materials}

The datasets used and analyzed during the current study are available from the corresponding author on reasonable request.

\section{Authors' contributions}

RK, JS, TE, GVG, and JB conceived the study, set up and conducted the regional anesthesia course for emergency physicians, and helped in implementing the technique and standard operating procedure on the emergency department. JS trained the ED nurses. JS and EvE collected the data and motivated their colleagues to participate. RK, JS, and EvE analyzed the data. RK and JS prepared the manuscript. All authors read and re-read the manuscript and provided valuable comments and suggestions.

\section{Ethics approval and consent to participate}

This study was deemed exempt from formal review by the regional ethics review board of Arnhem and Nijmegen, and approval was obtained from the institutional review board of the Radboud university medical center. Before the ultrasound-guided femoral nerve block or FICB was performed, oral informed consent was obtained as part of the standard operating procedure.

\section{Consent for publication}

Not applicable.

\section{Competing interests}

The authors declare that they have no competing interests.

\section{Publisher's Note}

Springer Nature remains neutral with regard to jurisdictional claims in published maps and institutional affiliations.

\section{Author details}

'Department of Anesthesiology, Pain and Palliative medicine, Radboud university medical center, Geert Grooteplein-Zuid 10, 6525 GA Nijmegen, The Netherlands. 'Emergency Department, Radboud university medical center, Geert Grooteplein-Zuid 10,6525 GA Nijmegen, The Netherlands. ${ }^{3}$ Emergency Department, Slingeland Hospital, Kruisbergseweg 25, 7009 BL Doetinchem, The Netherlands.

Received: 5 September 2017 Accepted: 15 February 2018

Published online: 02 March 2018

References

1. Todd KH, Ducharme J, Choiniere M, Crandall CS, Fosnocht DE, Homel P, et al. Pain in the emergency department: results of the pain and emergency medicine initiative (PEMI) multicenter study. J Pain. 2007:8(6):460-6.

2. Berben SA, Meijs TH, van Dongen RT, van Vugt AB, Vloet LC, Mintjes-de Groot JJ, et al. Pain prevalence and pain relief in trauma patients in the accident \& emergency department. Injury. 2008;39(5):578-85.

3. Chesters A, Atkinson P. Fascia iliaca block for pain relief from proximal femoral fracture in the emergency department: a review of the literature. Emerg Med J. 2014;31(e1):e84-7.
4. Mittal $R$, Vermani E. Femoral nerve blocks in fractures of femur: variation in the current UK practice and a review of the literature. Emerg Med J. 2014;31(2):143-7.

5. Unneby A, Svensson O, Gustafson Y, Olofsson B. Femoral nerve block in a representative sample of elderly people with hip fracture: a randomised controlled trial. Injury. 2017;48(7):1542-9.

6. Guay J, Parker MJ, Griffiths R, Kopp S. Peripheral nerve blocks for hip fractures. Cochrane Database Syst Rev. 2017;5:CD001159.

7. Beaudoin FL, Nagdev A, Merchant RC, Becker BM. Ultrasound-guided femoral nerve blocks in elderly patients with hip fractures. Am J Emerg Med. 2010;28(1):76-81.

8. Ritcey B, Pageau P, Woo MY, Perry JJ. Regional nerve blocks for hip and femoral neck fractures in the emergency department: a systematic review. CJEM. 2016;18(1):37-47.

9. Morrison RS, Magaziner J, Gilbert M, Koval KJ, McLaughlin MA, Orosz G, et al. Relationship between pain and opioid analgesics on the development of delirium following hip fracture. J Gerontol A Biol Sci Med Sci. 2003;58(1):76-81.

10. Mouzopoulos G, Vasiliadis G, Lasanianos N, Nikolaras G, Morakis E, Kaminaris M. Fascia iliaca block prophylaxis for hip fracture patients at risk for delirium: a randomized placebo-controlled study. J Orthop Traumatol. 2009;10(3):127-33.

11. Soeding PE, Sha S, Royse CE, Marks P, Hoy G, Royse AG. A randomized trial of ultrasound-guided brachial plexus anaesthesia in upper limb surgery. Anaesth Intensive Care. 2005:33(6):719-25.

12. Liu SS. Evidence basis for ultrasound-guided block characteristics onset, quality, and duration. Reg Anesth Pain Med. 2016;41(2):205-20.

13. Chin KJ, Chan V. Ultrasound-guided peripheral nerve blockade. Curr Opin Anaesthesiol. 2008:21(5):624-31.

14. Fuzier R, Tissot B, Mercier-Fuzier V, Barbero C, Caussade D, Mengelle F, et al. Evaluation of regional anesthesia procedure in an emergency department. Ann Fr Anesth Reanim. 2002;21(3):193-7.

15. Morrison RS, Dickman E, Hwang U, Akhtar S, Ferguson T, Huang J, et al. Regional nerve blocks improve pain and functional outcomes in hip fracture: a randomized controlled trial. J Am Geriatr Soc. 2016;64(12):2433-9.

16. Amini R, Kartchner JZ, Nagdev A, Adhikari S. Ultrasound-guided nerve blocks in emergency medicine practice. J Ultrasound Med. 2016;35(4):731-6.

17. Beaudoin FL, Haran JP, Liebmann O. A comparison of ultrasound-guided three-in-one femoral nerve block versus parenteral opioids alone for analgesia in emergency department patients with hip fractures: a randomized controlled trial. Acad Emerg Med. 2013;20(6):584-91.

18. Farrar JT, Berlin JA, Strom BL. Clinically important changes in acute pain outcome measures: a validation study. J Pain Symptom Manag. 2003;25(5):406-11.

19. Groot L, Dijksman LM, Simons MP, Zwartsenburg MM, Rebel JR. Single fascia iliaca compartment block is safe and effective for emergency pain relief in hip-fracture patients. West J Emerg Med. 2015;16(7):1188-93.

20. Dochez E, van Geffen GJ, Bruhn J, Hoogerwerf N, van de Pas H, Scheffer G. Prehospital administered fascia iliaca compartment block by emergency medical service nurses, a feasibility study. Scand J Trauma Resusc Emerg Med. 2014;22:38

21. Gozlan C, Minville V, Asehnoune K, Raynal P, Zetlaoui P, Benhamou D. Fascia iliaca block for femoral bone fractures in prehospital medicine. Ann $\mathrm{Fr}$ Anesth Reanim. 2005:24(6):617-20.

22. Haines L, Dickman E, Ayvazyan S, Pearl M, Wu S, Rosenblum D, et al. Ultrasound-guided fascia iliaca compartment block for hip fractures in the emergency department. J Emerg Med. 2012;43(4):692-7.

23. Wu JJ, Lollo L, Grabinsky A. Regional anesthesia in trauma medicine Anesthesiol Res Pract. 2011:2011:713281.

24. van Geffen GJ, Bruhn J, Hoogerwerf N, Slagt C. The journey should start at home. Int Emerg Nurs. 2016:25:76.

25. Riddell M, Ospina M, Holroyd-Leduc JM. Use of femoral nerve blocks to manage hip fracture pain among older adults in the emergency department: a systematic review. CJEM. 2016;18(4):245-52. 